\title{
Analysis of a UAV that can Hover and Fly Level
}

\author{
Ferit Çakıcı and M. Kemal Leblebicioğlu \\ Middle East Technical University, Department of Electrical and Electronics Department, 06800 Ankara, Turkey
}

\begin{abstract}
In this study, an unmanned aerial vehicle (UAV) with level flight, vertical take-off and landing (VTOL) and mode-changing capability is analysed. The platform design combines both multirotor and fixed-wing (FW) conventional airplane structures and control surfaces; therefore, named as VTOL-FW. The aircraft is modelled using aerodynamical principles and linear models are constructed utilizing small perturbation theory for trim conditions. The proposed method of control includes implementation of multirotor and airplane mode controllers and design of an algorithm to transition between modes in achieving smooth switching manoeuvres between VTOL and FW flight. Thus, VTOL-FW UAV's flight characteristics are expected to be improved by enlarging operational flight envelope through enabling mode-transitioning, agile manoeuvres and increasing survivability. Experiments conducted in simulation and real world environments show that, VTOL-FW UAV has both multirotor and airplane characteristics with extra benefits in an enlarged flight envelope.
\end{abstract}

\section{Introduction}

Innovative aerial vehicles have proved themselves in military and civil areas of different applications over a hundred years, by enhancing their capabilities over time, and adapting to new mission requirements. UAVs offer a unique set of advantages compared to piloted aircrafts with smaller, safer and lighter platforms. Future UAVs are expected to perform much more extended missions with higher manoeuvrability and higher degrees of autonomy.

Different capabilities like VTOL, hover, level flight, mode switching between hover and level modes, high endurance, long range and mechanical simplicity are expected from UAV platforms whenever mission requires. Comparison of capabilities of different types of UAV platforms (Table 1) provides insight about its mission profile. When VTOL and hovering are required, then rotary-wing aircraft such as helicopters, multirotors, ducted fans, tiltrotors and tailsitters are most optimal. However, if level flight, endurance or range is of priority, then a fixed-wing airplane type will most likely be preferred due to efficiency. When all of these features are desired, then VTOL-FW platforms becomes the best option, as an in-between platform among others. VTOL capability removes the need for runway or launch/recovery equipment and provides flexibility to operate in any theatre, whereas level flight capability allows efficient range and endurance flight. An aerial vehicle designed to possess the strengths of both a rotary and fixed-wing aircraft will have both of the advantages in one platform, with acceptable trade-offs in some capabilities.
Table 1. Comparison of UAV platform types.

\begin{tabular}{|c|c|c|c|c|}
\hline Capability & $\begin{array}{c}\text { Multi- } \\
\text { rotor }\end{array}$ & $\begin{array}{c}\text { Heli- } \\
\text { copter }\end{array}$ & Airplane & $\begin{array}{c}\text { VTOL- } \\
\text { FW }\end{array}$ \\
\hline VTOL & Good & Good & Bad & Good \\
\hline Hover & Good & Good & Bad & Good \\
\hline Level Flight & $\mathrm{Bad}$ & $\mathrm{Bad}$ & Good & Good \\
\hline $\begin{array}{c}\text { Mode- } \\
\text { Switching }\end{array}$ & $\mathrm{Bad}$ & $\mathrm{Bad}$ & $\mathrm{Bad}$ & Good \\
\hline Endurance & $\mathrm{Bad}$ & $\mathrm{Good}$ & Good & Neutral \\
\hline Range & $\mathrm{Bad}$ & $\mathrm{Bad}$ & Good & Neutral \\
\hline Simplicity & $\mathrm{Good}$ & $\mathrm{Bad}$ & Good & Neutral \\
\hline
\end{tabular}

Available studies [1-5] in VTOL-FW UAV platform category include tailsitters, tiltrotors and tiltwings. Although all of these platforms can perform hover and level flight, the main difference comes from the method of transition method (Table 2) between flight modes. These types of platforms suffer from difficult transition manoeuvres that operate the aircraft out of trim conditions and susceptibility to disturbances in transitions. However, a hybrid VTOL-FW platform asserted in this study, allows smooth transitions by being operated in an enlarged flight envelope.

Transition maneuvers between hover and level flight is of primary concern for VTOL aircrafts that are capable of level flight. T-wing tailsitter UAV with two counter rotating propellers was one of the pioneering studies; Stone [6] has developed a flight control system including low-level and mid-level guidance controllers, utilizing 
linear quadratic regulator and classical controllers, which were verified in flight tests [3]. Kubo [7] showed that a tailsitter UAV could achieve transitions between level flight and hover in shorter time using slats and flaps by using an optimal controller. Hogge [8] demonstrated transition maneuvers of a UAV with only one propulsion system using control surfaces. Tumble-stall maneuvers are implemented in achieving transitions by [5], [9] and [10] utilizing dynamic inversion methods. A state machine is designed by [11] and [12] for transitions between the flight modes, where the states were defined as hover, level, hover-to-level and level-to-hover. Backstepping control technique is studied by [2] for a coaxial-rotor tailsitter UAV and successfully simulated hover, level flight and transitions. Although available studies in this field are successfully implemented on different platform types, an aircraft that has physically separated multirotor and airplane control surfaces is not examined in demonstrating transition maneuvers.

Table 2. VTOL-FW UAV platforms' transition methods.

\begin{tabular}{|c|c|l|}
\hline Photograph & Type & \multicolumn{1}{c|}{ Transition Method } \\
\hline & Tailsitter & $\begin{array}{l}\text { Tilts fuselage by control } \\
\text { surfaces through stalling the } \\
\text { aircraft. }\end{array}$ \\
\hline & Tiltrotor & $\begin{array}{l}\text { Tilts fuselage by tilting rotors } \\
\text { that stalls wings. }\end{array}$ \\
\hline & Tiltwing & $\begin{array}{l}\text { Tilts wings that operates in } \\
\text { stall region, while the fuselage } \\
\text { remains parallel to Earth. }\end{array}$ \\
\hline
\end{tabular}

In this study, design, analysis and implementation of control system of a VTOL aircraft with level flight capability is considered. The proposed method includes implementation of multirotor and airplane controllers and design of an algorithm to switch between them in achieving transitions between VTOL and FW flight modes. Thus, VTOL-FW UAV's flight characteristics are expected to be improved by enabling agile maneuvers, increasing survivability, providing redundancy and exploiting full flight envelope capabilities.

\section{VTOL-FW UAV platform}

VTOL-FW UAV platform is constructed by multirotor modification of a model airplane (Figure 1). Then, the platform is converted into an UAV by adding an autonomous flight controller (Pixhawk) and sensors like GPS, magnetometers, accelerometers, gyros, pitot-static system as in [13].

Main components of the aircraft (Figure 2) contribute to forces and moments acting on the vehicle in flight. Fuselage causes drag in negative direction of airflow, caused by linear motion. FW propulsion system (Prop.0) provides thrust to balance drag, while main wing provides lift to overcome gravitational force and ailerons, rudder and elevator provide roll, pitch and yaw motions as in a conventional airplane. VTOL propulsion systems (Prop.1-4) provide lift, roll, pitch and yaw motions by changing the rotational speeds of the propellers, as in a multirotor.

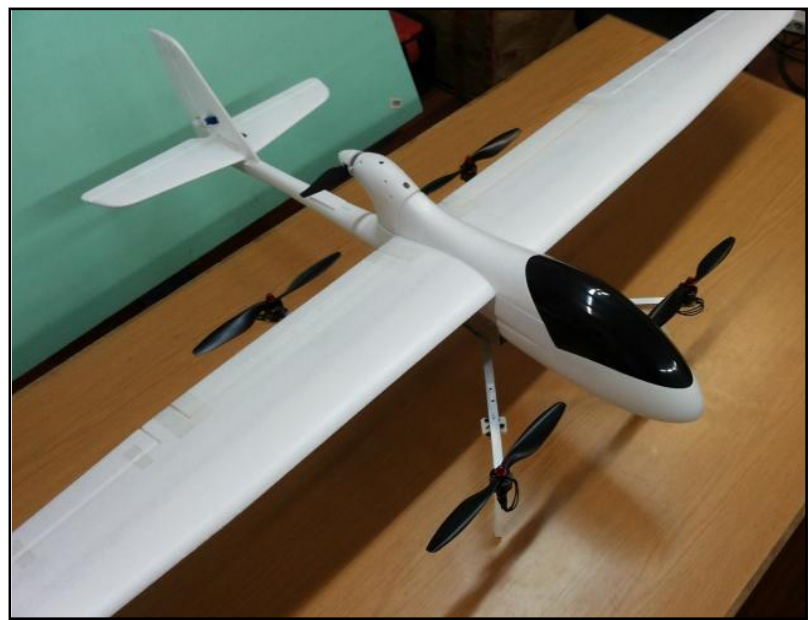

Figure 1. VTOL-FW UAV Platform.

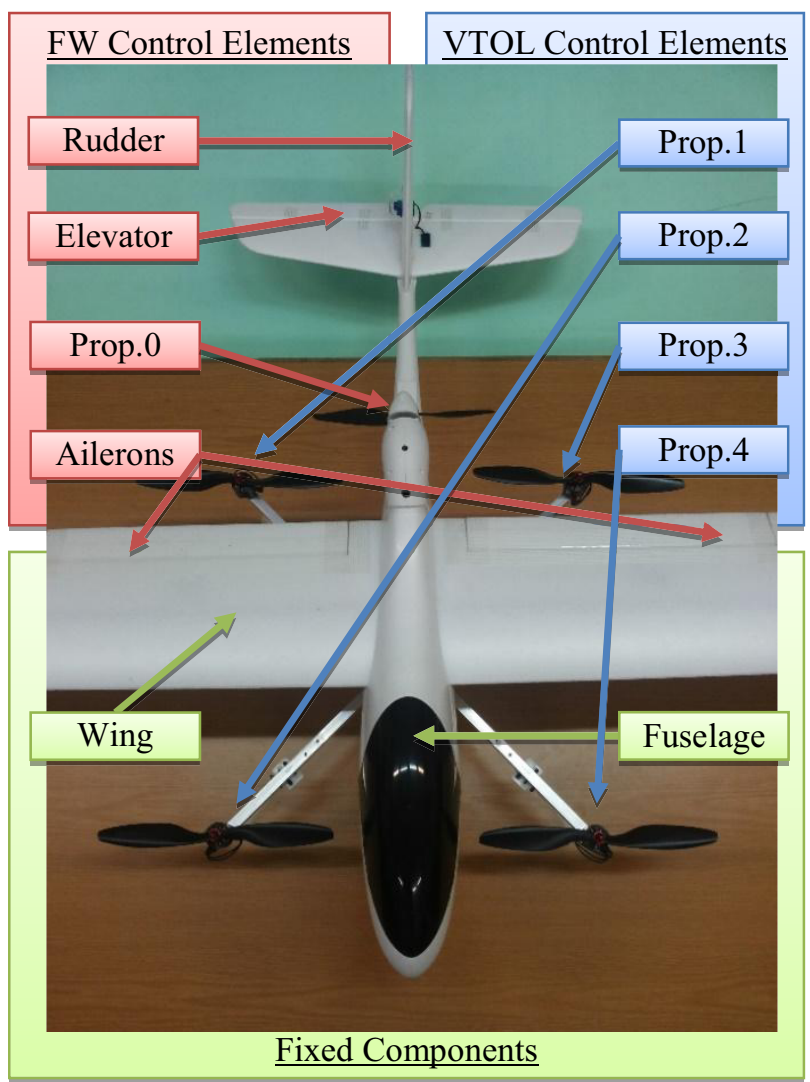

Figure 2. Control Elements of VTOL-FW UAV.

Flight mode of VTOL-FW UAV is determined according to vertical and horizontal velocities of the aircraft in an enlarged flight envelope (Figure 3), that covers both VTOL and FW regions. When both of the vertical and horizontal velocities are small in magnitude, the aircraft operates in VTOL mode with VTOL control elements activated. As horizontal velocity is increased the aircraft enters the FW mode by enabling FW control elements. Intersectional region is used for switching 
between VTOL and FW modes, by changing active control elements.

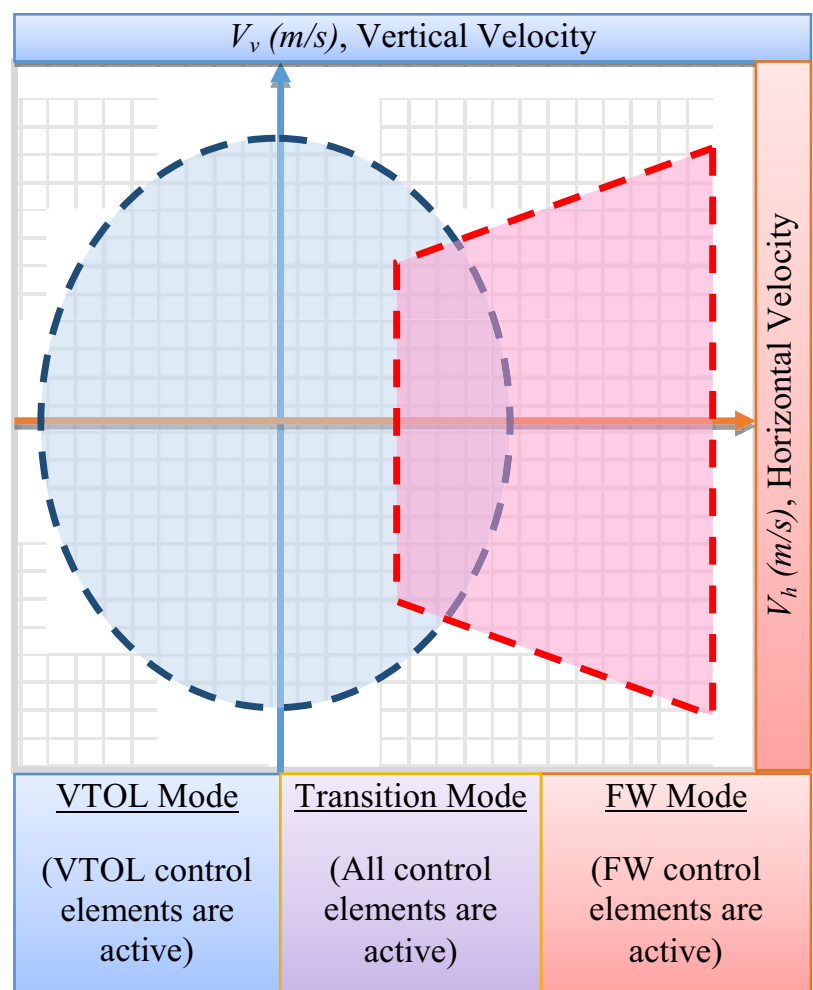

Figure 3. Flight envelope of VTOL-FW UAV platform.

\section{Mathematical modelling}

The complexity of dynamics of aerial vehicles, makes obtaining accurate mathematical models for a large portion of flight envelope a difficult problem. VTOL-FW UAV platform is modelled by using the real physical specifications of the aircraft in a MATLAB graphical user interface environment that is specifically developed for the preliminary design, analysis, control system design, mission planning and flight simulations of aircrafts. Initially, every main component like fuselage, wings, control surfaces and propellers are modelled using aerodynamical principles stated by Çakıcı [14], for the whole flight envelope including post-stall conditions. Then, these model's outputs are combined considering aircraft's geometry in calculating total forces $\left({ }^{B} F\right)$ and moments $\left({ }^{B} M\right)$. Equations of motion are formed as a set of nonlinear equations (1), using Newton's 2nd law of forces, Euler's formula for moments and kinematic relationships defined in body frame.

$$
\dot{x}=f(x, u, t)=f\left({ }^{B} F(x, u),{ }^{B} M(x, u), t\right)
$$

where $\mathrm{x}$ : state variables, $\mathrm{u}$ : control variables, t: time.

\section{Linear analysis}

Linear analysis of VTOL-FW UAV is performed by examining stability and controllability of the linearized system dynamics for trim states.

\subsection{Trimming}

A trim state is defined as the equilibrium point, where the rates of the aerodynamic state variables are zero, when the resultant forces and moments are in balance. The trimming problem concerns the determination of control commands $\left[u_{\text {rol }}, u_{\text {pit }}, u_{\text {yaw }}, u_{\text {th }}\right]$, which maps to control variables $\left[\theta_{\text {ail }}, \theta_{\text {ele }}, \theta_{\text {rud }}, \Omega_{0}, \Omega_{1}, \Omega_{2}, \Omega_{3}, \Omega_{4}\right], \quad$ and aerodynamical variables $[u, v, w, p, q, r, \phi, \theta]$ that are required to hold the aircraft in equilibrium for a set of prescribed variables $\left[\psi, x_{\varepsilon}, y_{\theta}, z_{\varepsilon}\right]$. The trim conditions are obtained by solving a constrained optimization problem, defined as:

$$
\begin{aligned}
& \text { Minimize }\left\|x_{a}^{\prime}\right\| \text { for } x_{p}, u \\
& \text { subject to } x_{a}^{\prime}=f\left(x_{a}, x_{p}, u\right) \text {, given } x_{p}^{\prime}
\end{aligned}
$$

where $x_{a}=[u, v, w, p, q, r, \phi, \theta], x_{p}=\left[\psi, x_{\varepsilon}, 0, z_{\varepsilon}\right]$, $u=\left[u_{\text {rol }}, u_{\text {pit }}, u_{\text {yaw }}, u_{\text {thr }}\right]$ and $f\left(x_{\alpha}, x_{p}, u\right)$ are the equations of motion.

Inspection of all prescribed operation points reveals that the trim condition could not be established for all of the points of interest, which reveals the limits of the flight envelope for each flight mode (Figure 4). An important observation is that the trim conditions of VTOL and FW flight modes intersect at level velocities between 12 and $16 \mathrm{~m} / \mathrm{s}$. This intersection region is used for transition maneuvers between modes. On the other hand, operation the in intersection region of the enlarged flight envelope allows inherent redundancy in control, which could save the UAV from crash due to failure of a control element, through switching to the other mode.

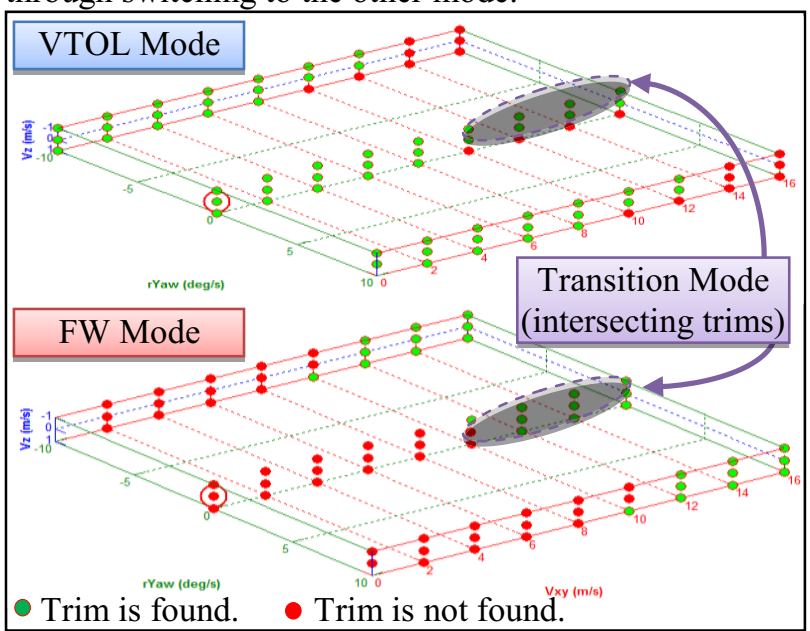

Figure 4. Trim conditions of VTOL-FW UAV.

Analysis of power requirements for an aircraft is important for achieving efficient flight. Assuming power dissipated on control surface servos is negligible and having lossless motors, electronic speed controllers, the major power consuming elements can be considered as propellers. Calculations of power required to fly (Figure 5) show that VTOL-FW UAV power consumption is similar to a comparable multirotor for hover. As level velocity is increased, multirotor power consumption increases due to the drag of the fuselage. As for VTOLFW UAV in VTOL mode, power requirement decreases as wings start to provide lift. The required power starts to increase after some point since more lift means more 
moment provided by aerodynamically surfaces, where VTOL propellers consume more power in struggling with elevator moment. The steep increase in the power in VTOL mode, as the velocity is increased, is one of the major reasons of the need to transition to the FW mode.

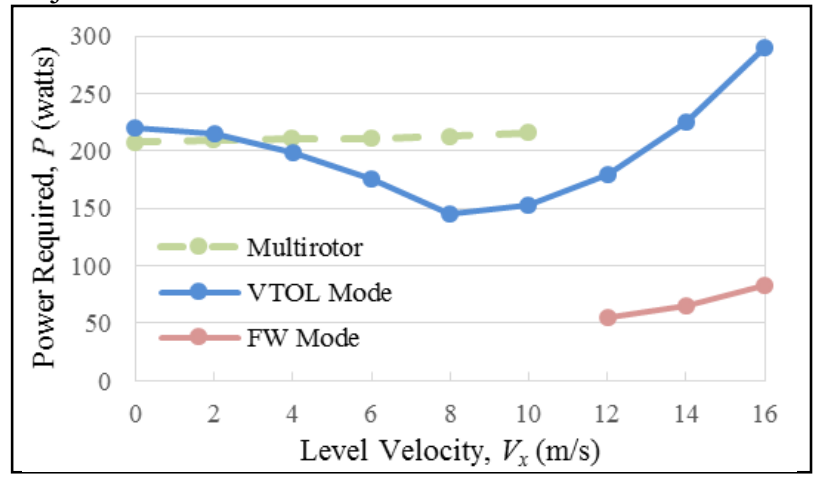

Figure 5. Power requirements of VTOL and FW modes.

\subsection{Stability}

Stability analysis (Figure 6) of the linearized dynamical system of VTOL-FW UAV shows that the aircraft is unstable in both modes. When a measure of stability is defined as the distance of the largest unstable pole in the right-hand s-plane to marginal stability line, the aircraft becomes more unstable in VTOL mode and less unstable in FW mode, as the horizontal velocity is increased. Analysis of the aircraft's dynamic modes proves that the aircraft demonstrates similar characteristics to an airplane in FW mode and to a multirotor in VTOL mode. Also, the controllability analysis shows that the aircraft is controllable in all of the trim points of both modes, which makes the controller design of the system possible. Eventually, stability analysis shown that VTOL-FW UAV demonstrates common aircraft characteristics in FW mode, and common multirotor characteristics with benefits in VTOL mode.

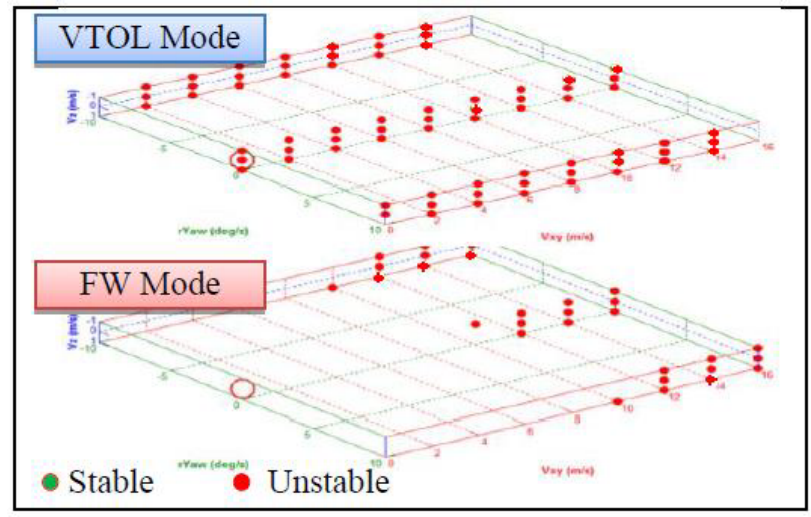

Figure 6. Stability of VTOL and FW modes.

\section{Control system}

A closed loop-control system is expected to stabilize the system, reject disturbances, reduce sensitivity to parameter variations, track reference, provide robustness to uncertainties, and be implementable for real world applications. Additionally, VTOL-UAV control system should make the aircraft follow guidance commands.
A control system architecture (Figure 7) is proposed that controls the aircraft in different flight modes. The inputs of the control system are obtained from guidance commands, and the outputs are defined as $\left[u_{\text {rol }}, u_{\text {pit }}, u_{\text {yaw }}, u_{\text {thr }}\right]$ which tell the aircraft to roll, pitch, yaw or change throttle, regardless of the active operation mode, which are then transformed into control element's commands through a control mixer.

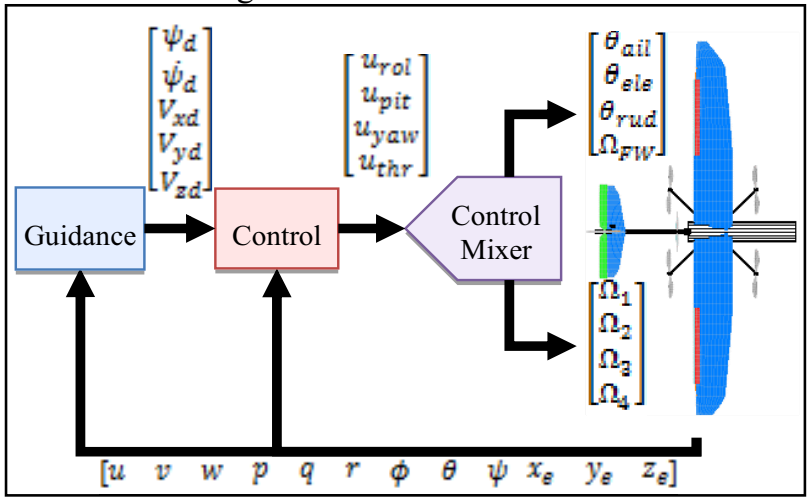

Figure 7. Control system architecture of VTOL-FW UAV.

\section{Flight}

A flight course of desired waypoints (Figure 8), forming a rectangle, is planned for flight testing. Initiating flight from waypoint 1 , the aircraft took off vertically in VTOL mode and started ascending towards waypoint 2. After reaching waypoint 2 , level velocity is increased in order to reach waypoint 3 . As the velocity is increased further, the autopilot changed the mode to FW for level flight. When the aircraft approaches to waypoint 3, the autopilot changed the mode to VTOL again and the final waypoint is reached by vertical landing.

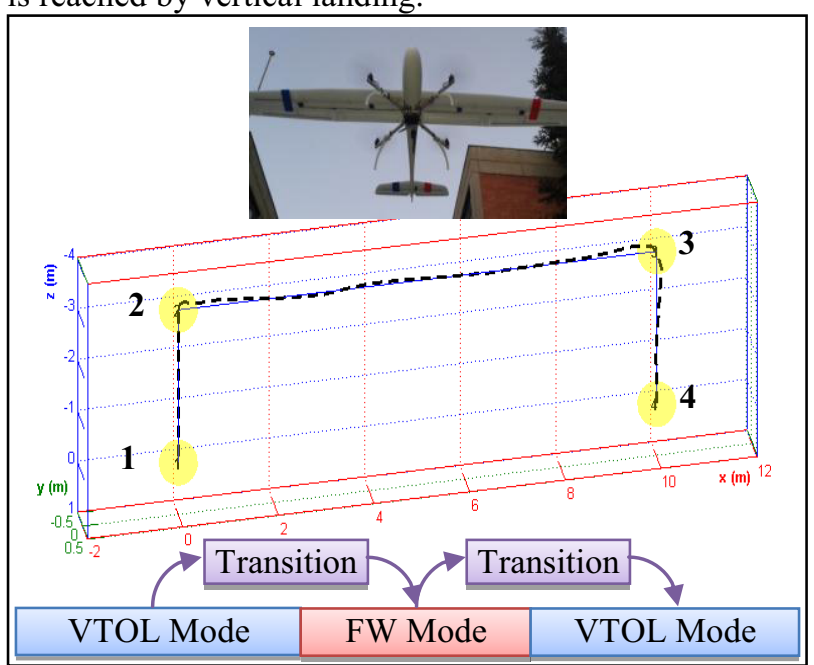

Figure 8. Flight experiment of VTOL-FW UAV.

The simulation results (Table 3 ) show that the aircraft completes waypoints (Figure 8) in shorter time with less power required when in "auto" mode, which automatically changes modes, compared to sole VTOL mode. The difference comes from the efficient flight operation through utilization of FW mode in auto so that the lift is mainly generated by the wings, instead of propellers of VTOL mode. These results prove the 
necessity and benefit of using different modes in an aircraft that can hover and fly level.

Table 3. Flight simulation results.

\begin{tabular}{|c|c|c|}
\hline Mode & Flight Time (s) & Average Power (W) \\
\hline VTOL & 6.83 & 256.47 \\
\hline $\begin{array}{c}\text { Auto } \\
\text { (VTOL-FW) }\end{array}$ & 5.21 & 163.81 \\
\hline
\end{tabular}

\section{Conclusion}

In this study, design and analysis of a UAV with VTOL, hover, level flight and mode switching capabilities, VTOL-FW, is considered. The aircraft demonstrates distinct flight characteristics of a multirotor and a fixedwing airplane on the same platform. The aircraft is modelled for analysis, simulation and controller design phases. Different PID controllers tuned for VTOL and FW modes performed well in flying the aircraft like a multirotor and airplane. Transitions between these modes are achieved through mode switching by applying smoothed control commands to control elements of different phases of flight.

One of the major problems in designing an air vehicle with VTOL and level flight capabilities is the location of center of gravity. An airplane requires center of gravity in front of the aerodynamical center, and a multirotor requires center of gravity close to aerodynamical center. Thus, this problem becomes a trade-off between better hover and better level flight. The aircraft fuselage remains mostly parallel to the Earth's surface in a large portion of its flight envelope. This allows both VTOL and FW mode flight envelopes intersect with each other, which makes the transition between these modes easier by having close trim state conditions. Also, the power consumption of VTOL-FW UAV in different modes reveals the necessity of switching modes for achieving efficient flight.

Simulation and real-world flight experiments proved the applicability of the proposed platform. VTOL-FW UAV is expected to find usage areas in missions requiring VTOL, hover and efficient level flight without the need for a runway and launch-recovery equipment.

\section{Acknowledgment}

This work is supported by The Scientific and Technological Research Council of Turkey (TÜBİTAK) under Grant 114E149 and 110E192.

\section{References}

1. T. Matsumoto, K. Kita, R. Suzuki, A. Oosedo, K. Go, Y. Hoshino, M. Uchiyama, 2010 IEEE ICRAA, 54$59,(2010)$

2. X. Wang, H. Lin, "Design and Control for RotorFixed Wing Hybrid Aircraft," Proceedings of the Institution of Mechanical Engineers, J. of A. 225-7, 831-847 (2011)

3. R. H. Stone, P. Anderson, C. Hutchison, A. Tsai, P. Gibbens, K. C. Wong, J. of A, 45-2, 673-685 (2008)

4. M. Hochstenbach, C. Notteboom, M. S. thesis, Ku Leuven Univ., (2014)

5. W. E. Green, P. Y. Oh, Proceedings of the 2005 IEEE/ASME International Conference on Advanced Intelligent Mechatronics, 693-698 (2005)

6. R. H. Stone, Institution of Mechanical Engineers, Part G: J. of A. E., 218-6 417-433 (2004)

7. D. Kubo, Proceedings of 25th International Congress of the Aeronautical Sciences, (2006)

8. J. V. Hogge, M.S. thesis, Dept. of Mechanical Engineering, Brigham Young Univ. (2008)

9. N. Anathkrishnan, H. C. Shim, Idea Research and Development, (2008)

10. Y. Jung, D. H. Shim, J. of I. \& R. S., 65-1-4, $137-$ 152 (2011)

11. S. R. Osborne, M.S. thesis, Dept. of Electrical and Computer Engineering, Brigham Young Univ., (2007)

12. F. Çakıcı, M. K. Leblebicioğlu, J. of D. M. and S., 94, 335-345 (2011)

13. F. Çakıcı, H. Ergezer, U. Irmak, and M. K. Leblebicioğlu, "Coordinated guidance for multiple UAVs," Transactions of the Institute of Measurement and Control (TIMC), 2015.

14. F. Çakıcı, M.S. thesis, Dept. of Electrical and Electronics Engineering, Middle East Technical Univ., (2009) 